\title{
Applicability of Touch Sense Controllers Using Warm and Cold Sensations
}

\author{
Miwa Nakanishi ${ }^{1}$ and Sakae Yamamoto ${ }^{2}$ \\ ${ }^{1}$ Keio University, Fac. of Science \& Technology, Dept. of Administration Engineering, \\ Hiyoshi 3-14-1, Kohoku, Yokohama 223-8522, Japan \\ miwa_nakanishi@ae.keio.ac.jp \\ ${ }^{2}$ Tokyo University of Science, Fac. of Engineering, Dept. of Management Science, \\ 1-3 Kagurazaka, Shinjuku, Tokyo 163-8522, Japan \\ sakae@ms.kagu.tus.ac.jp
}

\begin{abstract}
At present, designing controllers considering the physical aspect, such as a user's muscle load or range of movement, and the cognitive aspect, such as easy handling or simple functions, has become a basic requirement, and many products have satisfied these aspects. Thus, conversely describing, because it is difficult to find differences among products using these aspects for customers, manufacturers are seeking ways to effectively appeal to users in the psychological aspect. A touch sense is expected to produce new interaction that meets our psychological satisfaction beyond visual and auditory senses. In this study, aiming at producing the reality effectively in user-system interaction, we examine the applicability of controllers with warm and cold sensation that match users' images of a change in system conditions. In particular, 1) we examine if there is a relationship between the cold or warm sensation that users feel at their fingertips and an image of changes in system conditions, and 2) we examine if a slider-type controller with warm and cold sensations helps a user's finger to slide correctly and naturally. Furthermore, on the basis of the results of this study, we propose some concepts for applying a touch sense to actual situations.
\end{abstract}

Keywords: Touch sense, Warm and cold sensations, System conditions.

\section{Introduction}

In an overwhelming majority of user-system interaction, controllers that use hands as a modality to input information from a user to a system are adopted. Human hands are good at detail work and have a fine touch sense [1]-[4]. With advancing technologies, many controllers, from conventional handles and levers to the latest mice and touch pads, have appeared, and much ergonomic design has been applied to them. At present, designing controllers considering the physical aspect, such as a user's muscle load or range of movement, and the cognitive aspect, such as easy handling or simple functions, has become a basic requirement, and many products have satisfied these aspects. Thus, conversely describing, because it is difficult to find differences among 
products that use these aspects for customers, manufacturers are seeking ways to effectively appeal to users in the psychological aspect.

Regarding user-system interaction, it is well known that information is input from a system to a user via eyes or ears and that from a user to a system via hands or legs. However, Gibson [5][6] found that touching an object with hands could produce complex and rich information; hence, hands are a very useful modality that enable a user to obtain information from and provide information to a system simultaneously. Moreover, Norman [7] referred to the importance of embedding "reality" in designing a tool, and Katz [8] suggested that a touch sense with hands could give a more impressive reality to a user than the other senses. Therefore, a touch sense is expected to produce new interaction that meets our psychological satisfaction beyond visual and auditory senses.

In an experimental study to examine the possibility of using controllers with impressive reality, Nakanishi, et al. [9] found that users could recognize roughness or smoothness of the surface of a simple switch by just pushing it with their index finger, and that there were relationships that users shared between some of the images of system conditions and the roughness or smoothness that users felt when touching the surface of switches. (For example, users commonly thought of "start up" by the switch with a smooth surface and "stop" by that with a rough surface.) Furthermore, Nakanishi, et al. [10] carried out an experiment in which users experienced the remote controller of an air conditioner to which the above findings were applied, and obtained the results that attaching roughness and smoothness to the surface of individual buttons on the controller so that each matched the users' images made them feel more comfortable and relieved.

In this study, focusing on two additional viewpoints, we explore the application of a touch sense to controllers. The first point focuses on the warm and cold sensations felt at the fingertips and examines the applicability of the sensation to controllers. The second point focuses on active finger movement as applied to recent mobile phones, and in particular, examines finger slide in controlling system conditions. This study aims at producing the reality effectively in user-system interaction, and examines the applicability of controllers with warm and cold sensations that match users' images of a change in system conditions. In particular, 1) we examine if there is a relationship between the cold or warm sensation that users feel at their fingertips and an image of changes in system conditions and 2) we clarify if a slider-type controller with warm and cold sensations helps a user's finger to slide correctly and naturally.

\section{First Experiment}

In the first experiment, we tried to clarify whether there is a common image on a change in system conditions that occurred when users felt a warm or cold sensation at their fingertip while it was sliding on the controller.

\subsection{Method}

Experimental apparatus. We prepared an oblong panel composed of three pieces of thermo modules (Peltier devices) covered with an aluminum plate on which a user's finger slid (Fig. 1). A piece of thermo module uses the Peltier effect, which states that 


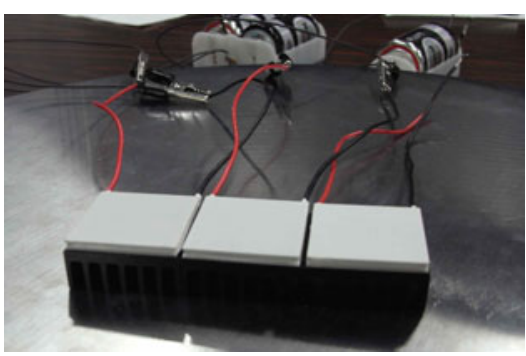

Fig. 1. Oblong panel composed of three pieces of thermo modules (Peltier devices) heat transfers from one side to another when a current is made to flow at two junctures and that it is possible to absorb and radiate heat by changing the current direction. Each part of the oblong panel was designed to be warmer or cooler than the normal temperature by connecting each of the three modules to one or more dry batteries. The room temperature was adjusted to $25-26^{\circ} \mathrm{C}$, so that the temperature of the panel surface was normally $25-26^{\circ} \mathrm{C}$ without passing a current.

In order that a subject sliding a finger on the oblong panel could feel a gradual change in temperature, different numbers of dry batteries were connected to each module. Table 1 shows six patterns of the temperature change. For example, when a subject slid a finger left to right on the oblong panel of pattern I-iii, he could feel a gradual temperature change, from cold to warm. The surface temperatures in Table 1 were measured using a surface thermometer ( $905-\mathrm{T} 2$, manufactured by Testo).

Table 1. Six patterns of arrangement of thermo modules

\begin{tabular}{|c|c|c|c|c|c|}
\hline \multicolumn{2}{|c|}{} & \multicolumn{3}{c|}{ Surface temperature $\left({ }^{\circ} \mathrm{C}\right)$} \\
\cline { 4 - 6 } & \multicolumn{2}{|c|}{} & Left & Center & Right \\
\hline \hline \multirow{3}{*}{$\begin{array}{c}\text { Increase } \\
\text { in temperature }\end{array}$} & Pattern I-i & Low - > Normal & 13 & 19 & 25 \\
\cline { 2 - 6 } & Pattern I-ii & Normal -> High & 26 & 37 & 48 \\
\cline { 2 - 6 } & Pattern I-iii & Low -> High & 19 & 27 & 37 \\
\hline \multirow{3}{*}{$\begin{array}{c}\text { Decrease } \\
\text { in temperature }\end{array}$} & Pattern II-i & High -> Normal & 48 & 36 & 25 \\
\cline { 2 - 6 } & Pattern II-ii & High -> Normal & 25 & 19 & 15 \\
\cline { 2 - 6 } & Pattern II-iii & Low - > Normal & 37 & 26 & 18 \\
\hline
\end{tabular}

Experimental task. The subjects positioned their index finger on the right or left side of the panel and slid it toward the opposite side (Fig. 2). They performed the task using the six patterns shown in Table 1 in two manners: left to right and right to left. To eliminate the order effect, the 12 experimental conditions were tested randomly.

After performing the tasks under each experimental condition, they answered questions about the relationship between the feeling of temperature change at their fingertip and the image of change in system conditions in the following manner. Twenty pairs of words repre-

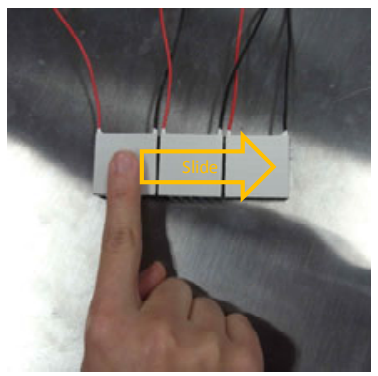

Fig. 2. Sliding the index finger on the thermo modules senting system conditions, which were composed on the basis of our previous study [9] (Table 2), were presented to them, and they selected the side of a pair word that corresponded more to the temperature change they experienced at their fingertip. For example, when they slid their finger left to right on the 
panel of pattern I-iii and

Table 2. Pair words representing system conditions

evaluated the experience on the image of a system condition of "Forward/Backward," if they felt that the experience matched with the image of "Backward," they selected it; however, if they felt that the experience matched with the image of "Forward," they selected it. While answering each pair word, they were allowed to repeatedly experience the

\begin{tabular}{|c|c|}
\hline Forward/Backward & Near/Far \\
\hline Up/Down & Stable/Instable \\
\hline Large/Small & Sharp/Blunt \\
\hline Fast/Slow & Light/Dark \\
\hline Long/Short & Soft/Hard \\
\hline High/Low & True/False \\
\hline Heavy/Light & Play/Pause \\
\hline Open/Close & Start/Stop \\
\hline Right/Left & On/Off \\
\hline Front/Back & Increase/Decrease \\
\hline
\end{tabular}
temperature change in the panel under an experimental condition.

Subject. The subjects included 16 students aging 22 to 26 . We obtained the informed consent of all participants.

\subsection{Results}

Fig. 3 shows the answers given for the pair words "Open/Close." The results indicate that most of the subjects thought of the image "Open" from the pattern of a temperature rise and the image "Close" from that of a temperature fall. However, we cannot find significant characteristics in the pattern where the temperature changed from high to normal or low to normal. Table 3 summarizes all results including the answers to the other pair words. In this table, the proportion that a left word was

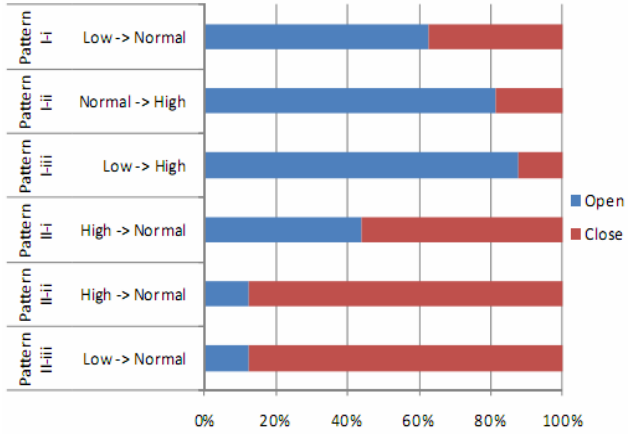

Fig. 3. Proportion of the answers given to the pair word "Open/Close" selected was represented with a negative value, and the proportion that a right word was selected was represented with a positive value. If the bias was symmetrical and the proportion was over $80 \%$, an asterisk was placed in the right row. As this table shows, the images of system conditions represented by all pair words except "Stable/Instable" and "Sharp/Blunt" were related to the temperature change. Moreover, it was confirmed that for a temperature change from high to normal or low to normal, it was difficult to create an image. 
Table 3. Proportion of the answers given to all pair words

\begin{tabular}{|c|c|c|c|c|c|c|c|c|c|c|c|c|c|c|c|c|c|c|c|c|c|}
\hline \multirow[b]{2}{*}{ Pattern Ir-iii } & \multirow[b]{2}{*}{ Low $\rightarrow$ Normal } & \multicolumn{2}{|c|}{ 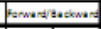 } & \multicolumn{2}{|c|}{ Us:osum } & \multicolumn{2}{|c|}{ 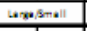 } & \multicolumn{2}{|c|}{ fun:Sow } & \multicolumn{2}{|c|}{ Langshen } & \multicolumn{2}{|c|}{ Hs how } & \multicolumn{2}{|c|}{ rawnest: } & \multicolumn{2}{|c|}{ senfelar. } & \multicolumn{2}{|c|}{ 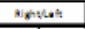 } & \multicolumn{2}{|c|}{ Fanfatad } \\
\hline & & $89 \%$ & $13 \%$ & $100 \%$ & $0 \%$ & $81 \%$ & $19 \%$ & 6339 & $38 \%$ & $69 \%$ & $31 \%$ & $100 \%$ & $0 \%$ & $50 \%$ & $50 \%$ & $81 \%$ & $19 \%$ & 88:5 & $13 \%$ & $63 \%$ & $38 \%$ \\
\hline Pattern $\|-\| \pi$ & $\mathrm{High} \rightarrow \mathrm{Normal}$ & & $44 \%$ & $75 \%$ & $25 \%$ & $443:$ & $56 \%$ & $56 \%$ & $44 \%$ & $50 \%$ & $50 \%$ & $56 \%$ & 445 & $25 \%$ & $75 \%$ & $63 \%$ & $38 \%$ & 56\%: & 445 & $56 \%$ & 445 \\
\hline Pastern III-i & $\mathrm{High}_{\mathrm{h}} \rightarrow \mathrm{Norms}$ & 8934 & $13 \%$ & 94\%: & $6 \%$ & $69 \%$ & $31 \%$ & $63 \%$ & $38 \%$ & $81 \%$ & 19\%: & 69:\% & $31 \%$ & $19 \% 6$ & 81\%: & 898 & 13\%: & 63\%: & $398 \%$ & $69 \%$ & 319 \\
\hline Pattern I-iii & Low $\rightarrow \mathrm{High}$ & $31 \%$ & $69 \%$ & $6 \%$ & $94 \%$ & $19 \%$ & $81 \%$ & $44 \%$ & $56 \%$ & $19 \%$ & $81 \%$ & $13 \%$ & 86: & $56 \%$ & $44 \times 5$ & $13 \%$ & $80 \%$ & $39 \%$ & 63\%: & $13 \%$ & 889 \\
\hline Partern l-ii & Normsl $\rightarrow \mathrm{High}$ & $50 \%$ & $50 \%$ & $50 \%$ & $50 \%$ & $63 \%$ & $38 \%$ & 4456 & $56 \%$ & $39 \%$ & $63 \%$ & $56 \%$ & $44 \%$ & $75 \%$ & $25 \%$ & 4458 & $56 \%$ & 56\%: & 443: & $56 \%$ & 448 \\
\hline Partern 1-i & Low $\rightarrow$ Norms & $25 \%$ & $75 \%$ & $0 \%$ & $100 \%$ & $6 \%$ & $94 \%$ & $38 \%$ & $63 \%$ & $31 \%$ & $69 \%$ & $19 \%$ & $81 \%$ & $50 \%$ & $50 \% 6$ & $13 \%$ & $80 \%$ & $25 \%$ & $75 \%$ & $25 \%$ & 75 \\
\hline & & & & & & & & & & & & & & & & & & & & & \\
\hline
\end{tabular}

\begin{tabular}{|c|c|c|c|c|c|c|c|c|c|c|c|c|c|c|c|c|c|c|c|c|c|}
\hline \multirow[b]{2}{*}{ Pattern |l-iii } & & \multicolumn{2}{|c|}{ Naw:Fer } & \multicolumn{2}{|c|}{ Sationuese } & \multicolumn{2}{|c|}{ Shesalun: } & \multicolumn{2}{|c|}{ Untourik } & \multicolumn{2}{|c|}{ Set/mard } & \multicolumn{2}{|c|}{ Frop foing } & \multicolumn{2}{|c|}{ neposuas } & \multicolumn{2}{|c|}{ saneystas } & \multicolumn{2}{|c|}{ on, on } & \multicolumn{2}{|c|}{ 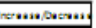 } \\
\hline & & $56 \%$ & $44 \%$ & $44 \%$ & $56 \%$ & $56 \%$ & $44: 5$ & 945: & $6 \%$ & 898 & $13 \%$ & $63 \%$ & $38 \%$ & 8985 & $13 \%$ & 81: & $19 \%$ & $100 \%$ & $0 \%$ & $94 \% /$ & $6 \%$ \\
\hline Pattern $\|-\| i$ & High $\rightarrow$ Normal & $50 \%$ & $50 \%$ & 81\% & $19 \%$ & $63 \%$ & $38 \% 6$ & $56 \%$ & $44 \%$ & $69 \%$ & $31 \%$ & $63 \%$ & $39 \%$ & $69 \%$ & $31 \%$ & $75 \%$ & $25 \%$ & $75 \%$ & $25 \%$ & 4456 & $56 \%$ \\
\hline Pattern II-i & $\mathrm{High}_{\mathrm{i}} \rightarrow$ Normal & $75 \%$ & $25 \%$ & $55 \%$ & $44 \%=$ & $56 \%$ & $44 \% 6$ & $81 \%$ & $19 \%$ & $94 \%$ & $6 \%$ & $75 \%$ & $25 \%$ & $100 \%$ & $0 \%$ & $88 \%$ & $13 \%$ & $94 \%$ & $6 \%$ & 94:3 & $6 \%$ \\
\hline Pattern I-iii & Low $\rightarrow \mathrm{High}_{1}$ & $13 \%$ & $89 \%$ & $69 \%$ & $31 \%$ & $25 \%$ & $75 \%$ & $6 \%$ & $94 \%$ & $19 \%$ & $81 \%$ & $56 \%$ & $44 \%$ & $6 \%$ & $94 \%$ & $13 \%$ & 89\% & $13 \%$ & 8986 & $0 \%$ & $100 \%$ \\
\hline Pattern l-ii & Normsl $\rightarrow$ High & $50 \%$ & $50 \%$ & $31 \%$ & $69 \%$ & $56 \%$ & $44 \%$ & $56 \%$ & $44 \%$ & $39 \%$ & $63 \%$ & $25 \%$ & $75 \%$ & $50 \%$ & $50 \%$ & $448 \mathrm{~g}$ & $56 \%$ & $50 \%$ & $50 \%$ & $56 \%$ & $44 \%$ \\
\hline Pastern I-i & Low $\rightarrow$ Norms & $31 \%$ & $69 \%$ & $56 \%$ & $44 \%=$ & $39 \%$ & 63\%: & 13\%: & $80 \%$ & $6 \%$ & $94 \%$ & $25 \%$ & $75 \%$ & $6 \%$ & $94 \%$ & $13 \%$ & 8096 & $0 \%$ & $100 \%$ & $0 \%$ & 1009 \\
\hline Biss & & & & & & & & & & & & & & & & & & & & & \\
\hline
\end{tabular}

\section{Second Experiment}

Next, we examined if the slider-type controller with warm and cold sensations that was designed on the basis of the results of the first experiment helped a user's correct and intuitive operation.

\subsection{Method}

Experimental apparatus. We prepared a mimic slide-type controller composed of five pieces of thermo modules (Peltier devices) covered with an aluminum plate. The number of dry batteries connected to each module and the direction of each current were fixed, as shown in Fig. 4, to set the temperature of each module to as shown in Fig. 5, so that a subject who slid a finger on the controller felt a temperature change (increase or decrease) from one side to another.

Experimental task. The subjects were given various words that indicated a change in system conditions (Table 4). They were asked to consider the given words as an

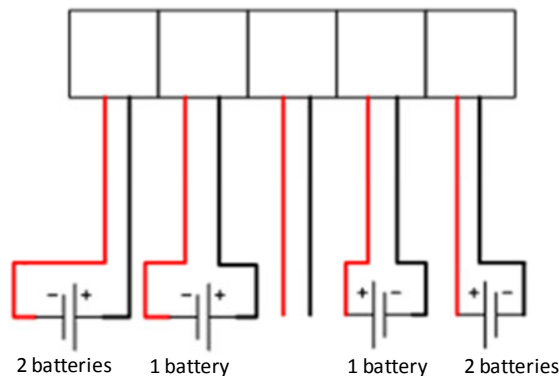

Fig. 4. Number of dry batteries connected to each module and the direction of each current

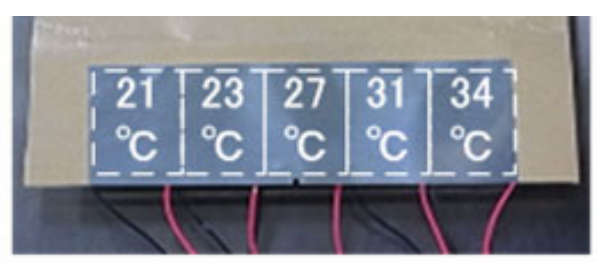

Fig. 5. Temperature of each module 
Table 4. Words meaning change in system conditions

\begin{tabular}{|c|c|c|c|}
\hline Up & A little up & A little down & Down \\
\hline Increase & A little increase & A little decrease & Decrease \\
\hline Open & A little Open & A little Close & Close \\
\hline Light & A little light & A little dark & Dark \\
\hline High & A little high & A little low & Low \\
\hline Large & A little large & A little small & Small \\
\hline Strong & A little strong & A little weak & Weak \\
\hline Forward & A little forward & A little backward & Backward \\
\hline
\end{tabular}

operation purpose and to operate the slider-type controller. These words were composed of eight types of system conditions and four grades for each type, which were selected from the pair words used in the first experiment. The subjects positioned their index finger on the center of the slider-type controller and slid it to the position of a temperature that they thought matched the image of the given word; that is, the operation purpose. For example, when they were given the operation purpose "A little open," they slid their finger from the center toward the right or left, feeling warm or cold, and stopped at the position they thought matched the image of the operation purpose. The subjects performed the task 64 times, with each of the above operation purposes, using two types of controllers, in which the temperature increased from left to right or decreased from left to right. To eliminate the order effect, the operation purposes and the types of controllers were given to the subjects randomly. After each trial, we recorded the position where their finger stopped.

Subject: The subjects included 21 students aging 22 to 26 . We obtained the informed consent of all participants.

\subsection{Results}

Fig. 6 shows the correlation between four grades of operation purposes concerning "Open/Close" and the temperature at which the subjects' finger stopped. From the results of the one-way ANOVA, we found a significant effect of the grades of the operation purposes on the temperature at which they stopped their finger $(\mathrm{F}=32.45, \mathrm{p}$ $<.05)$. This means that for the operation purpose "Open," their finger intuitively moved toward the direction of high temperature and

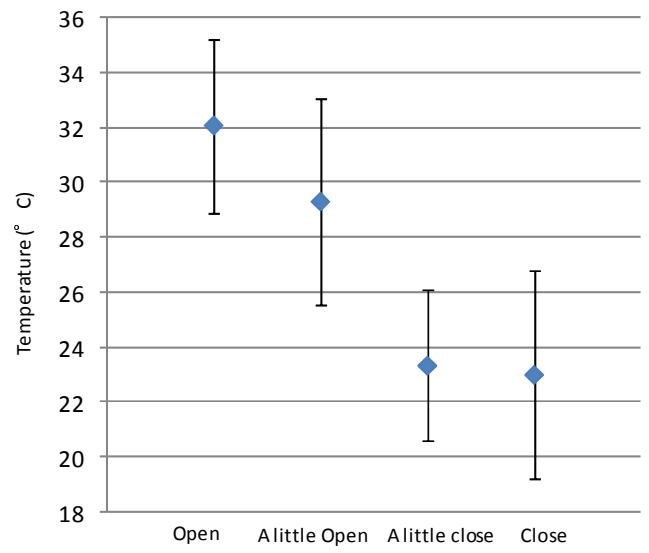

Fig. 6. Correlation between four grades of the operation purposes concerning "Open/Close" and the temperature at which the subjects' finger stopped 
for the operation purpose "Close," their finger intuitively moved toward the direction of low temperature. Only one subject showed the opposite reaction; thus, it was a common characteristic among the subjects. Moreover, the correlation coefficient 0.73 suggests that their finger stopped corresponding to the degree of the temperature. However, because the length of the finger movement was relative to the change in temperature in this experiment, it was understood that the correspondence of the degree between the system condition and the temperature described above was caused by both factors. Table 5 shows the correlation between the other operation purposes and the temperatures at which the subjects' finger stopped. The results indicated that the subjects' finger slid in common directions according to the increase or decrease in the temperature when they were given each operation purpose concerning the eight images of changing system conditions.

Table 5. Correlation between four grades of the operation purposes and the temperature at which the subjects' finger stopped

\begin{tabular}{|c|c|c|c|c|c|}
\cline { 5 - 6 } \multicolumn{2}{l}{} & F-value & ${ }^{*}<.05$ \\
\hline Up & A little up & A little down & Down & 77.55 & ${ }^{*}$ \\
\hline Increase & A little increase & A little decrease & Decrease & 67.13 & ${ }^{*}$ \\
\hline Open & A little Open & A little Close & Close & 32.45 & ${ }^{*}$ \\
\hline Light & A little light & A little dark & Dark & 42.74 & ${ }^{*}$ \\
\hline High & A little high & A little low & Low & 28.89 & ${ }^{*}$ \\
\hline Large & A little large & A little small & Small & 100.37 & ${ }^{*}$ \\
\hline Strong & A little strong & A little weak & Weak & 39.43 & ${ }^{*}$ \\
\hline Forward & A little forward & A little backward & Backward & 34.4 & ${ }^{*}$ \\
\hline
\end{tabular}

\section{Application}

This study's results suggest the applicability of the controller using warm and cold sensations to the following situations as more impressive user interfaces. First, we can transfer the application to controllers in a car. Although the drivers have to pay attention to the outside environment for safety, it is also necessary to operate the air conditioner, the audio, and the navigation in order to enjoy driving. A touch sense can be effectively applied to user interfaces that are difficult to pay attention to while operating the vehicle. For example, if warm and cold sensations is applied to the slider-type interface to control the volume of the audio, it might provide drivers with a more intuitive and enjoyable experience in the car. Second, it will be effective to use a touch sense for user interfaces to operate some functions of machines in industrial fields. In general, touch panels are installed in some of the latest industrial machines for setting the amount of work or patterns of processes. However, in the industrial fields, the work environment is often much different from that in offices, in terms of lighting and noise. In such cases, if workers can control the amount of work or patterns of processes by using a touch panel with warm and cold sensations that match their intuition, human error can be reduced and relief and comfort can be enhanced. 


\section{Conclusion}

In this study, aiming to effectively apply a touch sense to controllers in humansystem interaction, we examined the usefulness of applying warm and cold sensations that matched users' intuition to a slider-type interface in order to control system conditions. The results suggested the following ideas. First, 18 types of images concerning system conditions were related to warm and cold sensations common to users. Second, when users operated a slider-type controller with operation purposes such as "Open/Close" and "Up/Down," a warm or cold sensation navigated their finger in the direction that matched the operation purpose.

Furthermore, on the basis of the above results, we propose to apply warm and cold sensations to interfaces in private cars or industrial fields, in order to give users or workers a more error-free, comfortable, and enjoyable experience. While the hardware technologies related to a touch sense are progressing, it is important to explore how such technologies should be utilized to satisfy users' psychological aspects. This study focused on matching users' intuitive images, and obtained the basic data that can be useful to realize users' intrinsic comfort and satisfaction.

\section{References}

[1] Stevens, J.C., Green, B.G.: Temperature-touch interaction, Weber's phenomenon revisited. Sensory Processes 2, 206-219 (1978)

[2] Green, B.G.: Localization of thermal sensation, An illusion and synthetic heat. Percept Psychophys 22, 331-337 (1977)

[3] Stevens, J.C.: Thermal intensification of touch sensation, Further extensions of the Weber phenomenon. Sensory Processes 3, 240-248 (1974)

[4] Stevens, J.C., Choo, K.K.: Temperature sensitivity of the body surface over the life span. Somatosens Mot. Res. 15, 13-28 (1998)

[5] Gibson, J.J.: Observation on active touch. Psychol. Rev. 69, 477-490 (1962)

[6] Gibson, J.J.: The senses considered as perceptual systems. Houghton, Mifflin (1966)

[7] Norman, D.A.: The design of everyday things. Doubleday, New York (1990)

[8] Katz, D.: Der Aufbau der Tastwelt. Barth, Leiptig (1925); Krueger, L.E. (ed.) The world of touch. Erlbaum, Hillsdale (1989)

[9] Nakanishi, M., Yamamoto, S.: What Images Are Evoked by Tactile Feeling? -For Designing Control Interfaces Taking Tactile Feeling into Consideration. In: Proceedings of the Workshop on Tactile and Haptic Interaction, Tokyo, Japan, pp. 22-29 (2007)

[10] Nakanishi, M., Okada, Y., Yamamoto, S.: A Study on Effective Tactile Feeling of Control Panels for Electrical Appliances. In: Proceedings of the 13th International Conference on HCI (Human Computer Interaction), San Diego, USA, on CD-ROM (2009) 